\title{
RIEMANN COMPATIBLE TENSORS
}

\author{
CARLO ALBERTO MANTICA AND LUCA GUIDO MOLINARI
}

\begin{abstract}
Derdzinski and Shen's theorem on the restrictions posed by a Codazzi tensor on the Riemann tensor holds more generally when a Riemanncompatible tensor exists. Several properties are shown to remain valid in this broader setting. Riemann compatibility is equivalent to the Bianchi identity of the new "Codazzi deviation tensor", with a geometric significance. The general properties are studied, with their implications on Pontryagin forms. Examples are given of manifolds with Riemann-compatible tensors, in particular those generated by geodesic mapping. Compatibility is extended to generalized curvature tensors, with an application to Weyl's tensor and general relativity.
\end{abstract}

\section{INTRODUCTION}

The Riemann tensor $R_{i j k}{ }^{m}$ and its contractions, $R_{k l}=R_{k m l}{ }^{m}$ and $R=g^{k l} R_{k l}$, are the fundamental tensors to describe the local structure of a Riemannian manifold $\left(\mathscr{M}_{n}, g\right)$ of dimension $n$. In a remarkable theorem [10, 3] Derdzinski and Shen showed that the existence of a non trivial Codazzi tensor poses strong constraints on the structure of the Riemann tensor. Because of their geometric relevance, Codazzi tensors have been studied by several authors, as Berger and Ebin [1], Bourguignon 4], Derdzinski [8, 9], Derdzinski and Shen [10, Ferus [11, Simon 29]; a compendium of results is found in Besse's book [3]. Recently, we showed [22] that the Codazzi differential condition

$$
\nabla_{i} b_{j k}-\nabla_{j} b_{i k}=0
$$

is sufficient for the theorem to hold, and can be replaced by the more general notion of Riemann-compatibility, which is instead algebraic:

Definition 1.1. A symmetric tensor $b_{i j}$ is Riemann compatible ( $R$-compatible) if:

$$
b_{i m} R_{j k l}{ }^{m}+b_{j m} R_{k i l}{ }^{m}+b_{k m} R_{i j l}{ }^{m}=0 .
$$

With this requirement, we proved the following extension of Derdzinski-Shen's theorem:

Theorem 1.2. 22] Suppose that a symmetric R-compatible tensor $b_{i j}$ exists. Then, if $X, Y$ and $Z$ are three eigenvectors of the matrix $b_{r}{ }^{s}$ at a point of the manifold, with eigenvalues $\lambda, \mu$ and $\nu$, it is $R_{i j k l} X^{i} Y^{j} Z^{k}=0$ provided that both $\lambda$ and $\mu$ are different from $\nu$.

The concept of compatibility allows for a further extension of the theorem, where the Riemann tensor $R$ is replaced by a generalized curvature tensor $K$, and $b$ is

Date: 23 march 2012.

2010 Mathematics Subject Classification. Primary 53B20, Secondary 53B21.

Key words and phrases. Codazzi tensor, Riemann tensor, Riemann compatibility, generalized curvature tensor, geodesic mapping, Pontryagin forms. 
required to be $K$-compatible 22 .

This paper studies the properties of Riemann compatibility, and its implications on the geometry of the manifold. In section $2 R$-compatibility is shown to be equivalent to the Bianchi identity of a new tensor, the Codazzi deviation. In section 3 the irreducible components of the covariant derivative of a symmetric tensor are classified in a simple manner, based on the decomposition into traceless terms. This is of guidance in the study of different structures suited for $R$-compatibility. The general properties of Riemann compatibility are presented in section 4 . In section 5 several properties of manifolds in presence of a Riemann compatible tensor that were obtained by Derdzinsky-Shen and Bourguignon for manifolds with a Codazzi tensor, are recovered. In particular, it is shown that $R$-compatibility implies pureness, a property of the Riemann tensor introduced by Maillot that implies the vanishing of Pontryagin forms. Manifolds that display $R$-compatible tensors are presented in section 6 ; interesting examples are generated by geodesic mappings, that induce metric tensors that are $R$-compatible. Finally, in section $7, K$-tensors and $K$ compatibility are presented, with applications to the standard curvature tensors. In the end, an application to general relativity is mentioned, that will be discussed fully elsewhere.

\section{The Codazzi deviation tensor and R-Compatibility}

Since Codazzi tensors are Riemann compatible, for a non Codazzi differentiable simmetric tensor field $b$ it is useful to define its deviation from the Codazzi condition. This tensor solves an unexpected relation that generalizes Lovelock's identity for the Riemann tensor, and shows that Riemann compatibility is a condition for closedness of certain 2-forms.

Definition 2.1. The Codazzi deviation of a symmetric tensor $b_{k l}$ is

$$
\mathscr{C}_{j k l}=: \nabla_{j} b_{k l}-\nabla_{k} b_{j l}
$$

Simple properties are: $\mathscr{C}_{j k l}=-\mathscr{C}_{k j l}$ and $\mathscr{C}_{j k l}+\mathscr{C}_{k l j}+\mathscr{C}_{l j k}=0$.

The following identity holds in general, and relates the Bianchi differential combination for $\mathscr{C}$ to the Riemann compatibility of $b$ :

\section{Proposition 2.2.}

$$
\nabla_{i} \mathscr{C}_{j k l}+\nabla_{j} \mathscr{C}_{k i l}+\nabla_{k} \mathscr{C}_{i j l}=b_{i m} R_{j k l}{ }^{m}+b_{j m} R_{k i l}{ }^{m}+b_{k m} R_{i j l}{ }^{m}
$$

Proof.

$$
\begin{aligned}
& \nabla_{i} \mathscr{C}_{j k l}+\nabla_{j} \mathscr{C}_{k i l}+\nabla_{k} \mathscr{C}_{i j l}=\left[\nabla_{i}, \nabla_{j}\right] b_{k l}+\left[\nabla_{k}, \nabla_{i}\right] b_{j l}+\left[\nabla_{j}, \nabla_{k}\right] b_{i l} \\
& =b_{m l}\left(R_{i j k}{ }^{m}+R_{k i j}{ }^{m}+R_{j k i}{ }^{m}\right)+b_{i m} R_{j k l}{ }^{m}+b_{j m} R_{k i l}{ }^{m}+b_{k m} R_{i j l}{ }^{m}
\end{aligned}
$$

the first term vanishes by the first Bianchi identity.

Remark 2.3. The identity holds true if $b_{i j}$ is replaced by $b_{i j}^{\prime}=b_{i j}+\chi a_{i j}$, where $a_{i j}$ is a Codazzi tensor and $\chi$ a scalar field. Then: $\mathscr{C}_{j k l}^{\prime}=\mathscr{C}_{j k l}-\left(a_{k l} \nabla_{j}-a_{j l} \nabla_{k}\right) \chi$.

The deviation tensor is associated to the 2 -form $\mathscr{C}_{l}=\frac{1}{2} \mathscr{C}_{j k l} d x^{j} \wedge d x^{k}$. The closedness condition $0=D \mathscr{C}_{l}=\frac{1}{2} \nabla_{i} \mathscr{C}_{j k l} d x^{i} \wedge d x^{j} \wedge d x^{k}$ ( $D$ is the exterior covariant derivative) is the second Bianchi identity for the Codazzi deviation: $\nabla_{i} \mathscr{C}_{j k l}+$ $\nabla_{j} \mathscr{C}_{k i l}+\nabla_{k} \mathscr{C}_{i j l}=0$. This gives a geometric picture of Riemann compatibility: 
Theorem 2.4. $b_{i j}$ is Riemann compatible if and only if $D \mathscr{C}_{l}=0$.

Remark 2.5. The Codazzi deviation of the Ricci tensor is, by the contracted second Bianchi identity: $\mathscr{C}_{j k l}=: \nabla_{j} R_{k l}-\nabla_{k} R_{j l}=-\nabla_{m} R_{j k l}{ }^{m}$. For the Ricci tensor the identity (44) identifies with Lovelock's identity [18, for the Riemann tensor:

$$
\begin{aligned}
& \nabla_{i} \nabla_{m} R_{j k l}{ }^{m}+\nabla_{j} \nabla_{m} R_{k i l}{ }^{m}+\nabla_{k} \nabla_{m} R_{i j l}{ }^{m} \\
& \quad=-R_{i m} R_{j k l}{ }^{m}-R_{j m} R_{k i l}{ }^{m}-R_{k m} R_{i j l}{ }^{m} .
\end{aligned}
$$

A Veblen-like identity holds, that corresponds to (14) (For $b_{i j}=R_{i j}$ it specializes to Veblen's identity for the divergence of the Riemann tensor [20]):

\section{Proposition 2.6.}

$$
\begin{array}{r}
\nabla_{i} \mathscr{C}_{j l k}+\nabla_{j} \mathscr{C}_{k i l}+\nabla_{k} \mathscr{C}_{l j i}+\nabla_{l} \mathscr{C}_{i k j} \\
=b_{i m} R_{j l k}{ }^{m}+b_{j m} R_{k i l}{ }^{m}+b_{k m} R_{l j i}{ }^{m}+b_{l m} R_{i k j}{ }^{m}
\end{array}
$$

Proof. Write four equations (4) with cycled indices $i, j, k, l$ and sum up. Then simplify by means of the first Bianchi identity for the Riemann tensor and the cyclic identity $\mathscr{C}_{j k l}+\mathscr{C}_{k l j}+\mathscr{C}_{l j k}=0$.

\section{IRREDUCiBle COMPONENTS FOR $\nabla_{j} b_{k l}$ AND $R$-COMPATIBILITY}

We begin with a simple procedure to classify the $O(n)$ invariant components of the tensor $\nabla_{j} b_{k l}$. They will guide us in the study of $R$-compatibility.

If $b$ is the Ricci tensor, this simple construction reproduces the seven equations linear in $\nabla_{i} R_{j k}$, invariant for the $O(n)$ group, that are enumerated and discussed in Besse's treatise "Einstein Manifolds" [3].

For a simmetric tensor $b_{k l}$ with $\nabla_{j} b_{k l} \neq 0$, the tensor $\nabla_{j} b_{k l}$ can be decomposed into $O(n)$ invariant terms, where $\mathscr{B}_{j k l}^{0}$ is traceless $\left(\mathscr{B}_{j k}^{0}{ }^{j}=\mathscr{B}_{k j}^{0}{ }^{j}=0\right)$ [14, 17]:

$$
\begin{gathered}
\nabla_{j} b_{k l}=\mathscr{B}_{j k l}^{0}+A_{j} g_{k l}+B_{k} g_{j l}+B_{l} g_{j k} \\
A_{j}=\frac{(n+1) \nabla_{j} b^{m}{ }_{m}-2 \nabla_{m} b^{m}{ }_{j}}{n^{2}+n-2}, \quad B_{j}=-\frac{\nabla_{j} b^{m}{ }_{m}-n \nabla_{m} b^{m}{ }_{j}}{n^{2}+n-2}
\end{gathered}
$$

The traceless tensor can then be written as a sum of orthogonal components [18]:

$$
\mathscr{B}_{j k l}^{0}=\frac{1}{3}\left[\mathscr{B}_{j k l}^{0}+\mathscr{B}_{k l j}^{0}+\mathscr{B}_{l j k}^{0}\right]+\frac{1}{3}\left[\mathscr{B}_{j k l}^{0}-\mathscr{B}_{k j l}^{0}\right]+\frac{1}{3}\left[\mathscr{B}_{j l k}^{0}-\mathscr{B}_{l j k}^{0}\right]
$$

The orthogonal subspaces classify the $O(n)$ invariant equations that are linear in $\nabla_{j} b_{k l}$. The trivial subspace: $\nabla_{j} b_{k l}=0$. The subspace $\mathcal{I}$ (we follow Gray's notation, [13]) where $\mathscr{B}_{j k l}^{0}=0$ :

$$
\nabla_{j} b_{k l}=A_{j} g_{k l}+B_{k} g_{j l}+B_{l} g_{j k} .
$$

The complement $\mathcal{I}^{\perp}$ is characterized by $A_{j}, B_{j}=0$ i.e. $\nabla_{j} b_{k l}$ is traceless. This gives two invariant equations: $\nabla_{j} b^{j} l=0$, and $\nabla_{j} b^{m}{ }_{m}=0$. Since $\nabla_{j} b_{k l}=\mathscr{B}_{j k l}^{0}$, the structure of $\mathscr{B}^{0}$ specifies two orthogonal subspaces $\mathcal{I}^{\perp}=\mathcal{A} \oplus \mathcal{B}$. In $\mathcal{A}$ :

In $\mathcal{B}:$

$$
\nabla_{j} b_{k l}+\nabla_{k} b_{l j}+\nabla_{l} b_{j k}=0
$$

$$
\nabla_{j} b_{k l}-\nabla_{k} b_{j l}=0
$$


The subspace $\mathcal{I} \oplus \mathcal{A}$ contains tensors with traceless part $\nabla_{j} b_{k l}-A_{j} g_{k l}-B_{k} g_{j l}-B_{l} g_{j k}$ that solves the cyclic condition:

$$
\left[\nabla_{j} b_{k l}-\frac{1}{n+2}\left(\nabla_{j} b_{m}^{m}+2 \nabla_{m} b^{m}{ }_{j}\right) g_{k l}\right]+\text { cyclic }=0 .
$$

The subspace $\mathcal{I} \oplus \mathcal{B}$ contains tensors with traceless part that solves the Codazzi condition:

$$
\left[\nabla_{j} b_{k l}-\frac{1}{n-1}\left(\nabla_{j} b_{m}^{m}-\nabla_{m} b_{j}^{m}\right) g_{k l}\right]=\left[\nabla_{k} b_{j l}-\frac{1}{n-1}\left(\nabla_{k} b_{m}^{m}-\nabla_{m} b_{k}^{m}\right) g_{j l}\right]
$$

Accordingly, the Codazzi deviation tensor has the (unique) decomposition in irreducible components

$$
\mathscr{C}_{j k l}=\mathscr{C}_{j k l}^{0}+\lambda_{j} g_{k l}-\lambda_{k} g_{j l}, \quad \lambda_{j}=A_{j}-B_{j}=\frac{\nabla_{j} b^{m}{ }_{m}-\nabla_{m} b^{m}{ }_{j}}{n-1}
$$

where $\mathscr{C}^{0}$ is traceless. Eq.(4) becomes

$$
\begin{array}{r}
b_{i m} R_{j k l}{ }^{m}+b_{j m} R_{k i l}{ }^{m}+b_{k m} R_{i j l}{ }^{m}=\nabla_{i} \mathscr{C}_{j k l}^{0}+\nabla_{j} \mathscr{C}_{k i l}^{0}+\nabla_{k} \mathscr{C}_{i j l}^{0} \\
\quad+g_{i l}\left(\nabla_{j} \lambda_{k}-\nabla_{k} \lambda_{j}\right)+g_{j l}\left(\nabla_{k} \lambda_{i}-\nabla_{i} \lambda_{k}\right)+g_{k l}\left(\nabla_{i} \lambda_{j}-\nabla_{j} \lambda_{i}\right)
\end{array}
$$

There are only two orthogonal invariant cases:

- $\mathscr{C}_{j k l}^{0}=0$, then $b$ is $R$-compatible if and only if $\lambda$ is closed. If $b$ is the Ricci tensor, this requirement gives Nearly conformally symmetric $(N C S)_{n}$ manifolds, that were introduced by Roter 28 .

$-\nabla_{j} b^{m}{ }_{m}-\nabla_{m} b^{m}{ }_{j}=0$ then $b$ is $R$-compatible if and only if $\mathscr{C}=\mathscr{C}^{0}$ solves the second Bianchi dentity. If $b$ is the Ricci tensor, this corresponds to $\nabla_{j} R=0$.

Remark 3.1. The decomposition (10) for the deviation of the Ricci tensor turns out to be

$$
\mathscr{C}_{j k l}=-\frac{n-2}{n-3} \nabla_{m} C_{j k l}{ }^{m}+\frac{1}{2(n-1)}\left[g_{k l} \nabla_{j} R-g_{j l} \nabla_{k} R\right]
$$

where $C_{j k l}{ }^{m}$ is the conformal curvature tensor, or Weyl's tensor. In this case the $\lambda$ covector is closed.

\section{Riemann compatibility: general Properties}

The existence of a Riemann compatible tensor has various implications. A first one is the existence of a generalized curvature tensor. This leads to the generalization of Derdzinski-Shen theorem and other relations that were obtained for Codazzi tensors.

We need the definition, from Kobayashi and Nomizu's book [16]:

Definition 4.1. A tensor $K_{i j l m}$ is a generalized curvature tensor (or, briefly, a $K$-tensor) if it has the symmetries of the Riemann curvature tensor:

a) $K_{i j k l}=-K_{j i k l}=-K_{i j l k}$,

b) $K_{i j k l}=K_{k l i j}$,

c) $K_{i j k l}+K_{j k i l}+K_{k i j l}=0$ (first Bianchi identity).

It follows that the tensor $K_{j k}=:-K_{m j k}{ }^{m}$ is symmetric.

Theorem 4.2. If $b$ is $R$-compatible then $K_{i j k l}=: R_{i j p q} b^{p}{ }_{k} b^{q}$ is a K-tensor. 
Proof. a) For example: $K_{i j l k}=R_{i j r s} b_{l}{ }^{r} b_{k}{ }^{s}=R_{i j s r} b_{l}{ }^{s} b_{k}{ }^{r}=-R_{i j r s} b_{l}{ }^{s} b_{k}{ }^{r}=$ $-K_{i j k l}$. Property c) follows from (2): $K_{i j k l}+K_{j k i l}+K_{k i j l}=R_{i j r s} b_{k}{ }^{r} b_{l}{ }^{s}+$ $R_{j k r s} b_{i}{ }^{r} b_{l}{ }^{s}+R_{k i r s} b_{j}{ }^{r} b_{l}{ }^{s}=\left(R_{j i s}{ }^{r} b_{k r}+R_{k j s}{ }^{r} b_{i r}+R_{i k s}{ }^{r} b_{j r}\right) b_{l}{ }^{s}=0$. Property b) follows from c): $K_{i j k l}+K_{j k i l}+K_{k i j l}=0$. Sum the identity over cyclic permutations of all indices $i, j, k, l$ and use the symmetries a).

It is easy to see that a first Bianchi identity holds also for the last three indices: $K_{i j k l}+K_{i k l j}+K_{i l j k}=0$.

The next result remarks the relevance of the local basis of eigenvectors of the Ricci tensor. Another symmetric contraction of the Riemann tensor was introduced by Bourguignon [4]:

$$
\stackrel{\circ}{\mathrm{R}}_{i j}=: b^{p q} R_{p i j q} .
$$

Theorem 4.3. If $b$ is $R$-compatible then:

1) $b_{i m} R_{j}{ }^{m}-b_{j m} R_{i}{ }^{m}=0$,

2) $b_{i m}{\stackrel{\circ}{R_{j}}}^{m}-b_{j m} \stackrel{\circ}{R}_{i}^{m}=0$

Proof. The first identity is proven by transvecting (2) with $g^{k l}$. The second one is a restatement of the symmetry of the tensor $K_{i j}$.

Remark 4.4. A) Identies 1 and 2 are here obtained directly from $R$-compatibility. Bourguignon [4 obtained them from Weitzenböck's formula for Codazzi tensors, and Derdzinski and Shen 10, from their theorem.

B) As the symmetric matrices $b_{i j}, R_{i j}, \stackrel{\circ}{R}_{i j}$ commute, they share at each point of the manifold an orthonormal set of $n$ eigenvectors.

C) If $b^{\prime}$ is a symmetric tensor that commutes with a Riemann compatible $b$, then it can be shown that $R_{i j}^{\prime}=: b^{\prime p q} R_{p i j q}$ commutes with $b$.

Finally, this Veblen-type identity holds:

Proposition 4.5. If $b$ is $R$-compatible, then:

$$
b_{i m} R_{j l k}{ }^{m}+b_{j m} R_{k i l}{ }^{m}+b_{k m} R_{l j i}{ }^{m}+b_{l m} R_{i k j}{ }^{m}=0
$$

Proof. Write four equations (2) with cycled indices $i, j, k, l$ and sum up, and use the first Bianchi identity.

\section{Pure Riemann tensors and Pontryagin forms}

Riemann compatibility and nondegeneracy of the eigenvalues of $b$ imply directly that the Riemann tensor is pure and Pontryagin forms vanish.

We quote two results from Maillot's paper [19]:

Definition 5.1. In a Riemann manifold $\mathscr{M}_{n}$, the Riemann curvature tensor is pure if at each point of the manifold there is an orthonormal basis of $n$ tangent vectors $X(1), \ldots, X(n), X(a)^{i} X(b)_{i}=\delta_{a b}$, such that the tensors $X(a)^{i} \wedge X(b)^{j}=$ : $X(a)^{i} X(b)^{j}-X(a)^{j} X(b)^{i}, a<b$, diagonalize it:

$$
R_{i j}{ }^{l m} X(a)^{i} \wedge X(b)^{j}=\lambda_{a b} X(a)^{l} \wedge X(b)^{m}
$$

Theorem 5.2. If a Riemannian manifold has pure Riemann curvature tensor, then all Pontryagin forms vanish. 
Consider the maps on tangent vectors, built with the Riemann tensor,

$$
\begin{aligned}
& \omega_{4}\left(X_{1} \ldots X_{4}\right)=R_{i j a}{ }^{b} R_{k l b}{ }^{a}\left(X_{1}^{i} \wedge X_{2}^{j}\right)\left(X_{3}^{k} \wedge X_{4}^{l}\right), \\
& \omega_{8}\left(X_{1} \ldots X_{8}\right)=R_{i j a}{ }^{b} R_{k l b}{ }^{c} R_{m n c}{ }^{d} R_{p q d}{ }^{a}\left(X_{1}^{i} \wedge X_{2}^{j}\right) \cdots\left(X_{7}^{p} \wedge X_{8}^{q}\right), \\
& \ldots \ldots
\end{aligned}
$$

They are antisymmetric under exchange of vectors in the single pairs, and for cyclic permutation of pairs. The Pontryagin forms $26 \Omega_{4 k}$ result from total antisymmetrization of $\omega_{4 k}: \Omega_{4 k}\left(X_{1} \ldots X_{4 k}\right)=\sum_{P}(-1)^{P} \omega_{4 k}\left(X_{i_{1}} \ldots X_{i_{4 k}}\right)$ where $P$ is the permutation taking $(1 \ldots 4 k)$ to $\left(i_{1} \ldots i_{4 k}\right)$. $\Omega_{4 k}=0$ if two vectors repeat, intermediate forms $\Omega_{4 k-2}$ vanish identically.

Pontryagin forms on generic tangent vectors are linear combinations of forms evaluated on basis vectors.

If the Riemann tensor is pure, all Pontryagin forms on the basis of eigenvectors of the Riemann tensor vanish. For example, if $X, Y, Z, W$ are orthogonal: $\omega_{4}(X Y Z W)=\lambda_{X Y} \lambda_{Z W}\left(X^{a} \wedge Y^{b}\right)\left(Z_{b} \wedge W_{a}\right)=0$ and $\Omega_{4}(X Y Z U)=0$.

A consequence of the extended Derdzinski-Shen theorem 1.2 is the following:

Theorem 5.3. If a symmetric tensor field $b_{i j}$ exists, that is $R$-compatible and has distinct eigenvalues at each point of the manifold, then the Riemann tensor is pure and all Pontryagin forms vanish.

Proof. At each point of the manifold the symmetric matrix $b_{i j}(x)$ is diagonalized by $n$ tangent orthonormal vectors $X(a)$, with distinct eigenvalues. Since $b$ is $R$ compatible, theorem 1.2 holds and, because of antisymmetry of $R$ in first two indices:

$$
0=R_{i j}{ }^{k l} X(a)^{i} \wedge X(b)^{j} X(c)_{k}, \quad a \neq b \neq c .
$$

This means that all column vectors of the matrix $V(a, b)^{k l}=R_{i j}{ }^{k l} X(a)^{i} \wedge X(b)^{j}$ are orthogonal to vectors $X(c)$ i.e. they belong to the subspace spanned by $X(a)$ and $X(b)$. Because of antisymmetry in indices $k, l$, it is necessarily $V(a, b)=$ $\lambda_{a b} X(a) \wedge X(b)$, i.e. the Riemann tensor is pure.

This property has been checked by Petersen [27] in various examples with rotationally invariant metrics, by giving explicit orthonormal frames such that $R\left(e_{i}, e_{j}\right) e_{k}=$ 0 .

5.1. Two and three dimensional manifolds. Riemannian manifolds of dimension $n=2$ and $n=3$ are special, as the Riemann tensor is expressible in terms of the Ricci and metric tensors. Therefore, Riemann-compatibility and ensuing pureness of the Riemann tensor can be established by simple means.

$n=2) R_{j k l m}=R_{j l} g_{k m}-g_{j m} R_{k j}$. Explicit evaluation proves that any symmetric tensor $b$ is Riemann compatible.

$n=3) R_{j k l m}=g_{j l} R_{k m}+g_{k m} R_{j l}-g_{k l} R_{j m}-g_{j m} R_{k l}-\frac{R}{2}\left(g_{j l} g_{k m}-g_{j m} g_{k l}\right)$. Then, for any symmetric tensor $b$ it is:

$$
\begin{array}{r}
b_{i m} R_{j k l}{ }^{m}+b_{j m} R_{k i l}{ }^{m}+b_{k m} R_{i j l}{ }^{m}=g_{k l}\left(b_{j m} R_{i}{ }^{m}-b_{i m} R_{j}{ }^{m}\right) \\
+g_{i l}\left(b_{k m} R_{j}{ }^{m}-b_{j m} R_{k}{ }^{m}\right)+g_{j l}\left(b_{i m} R_{k}{ }^{m}-b_{k m} R_{i}{ }^{m}\right)
\end{array}
$$


Thus in $n=3$ the Ricci tensor is always $R$-compatible. Moreover, if $b$ commutes with the Ricci tensor, then $b$ is $R$-compatible. Since a symmetric tensor that commutes with the Ricci tensor can always be constructed, with arbitrarily chosen distinct eigenvalues, by theorem 5.3 we conclude:

Proposition 5.4. In Riemannian manifolds of dimension $n=2$ and $n=3$ the Riemann tensor is pure.

5.2. Quasi-constant curvature spaces. The same conclusions can be drawn in any dimension $n$ for quasi-constant curvature spaces. They were introduced by Chen and Yano $[5$ and are defined by a Riemann tensor with the following structure:

$$
R_{j k l m}=p\left[g_{m j} g_{k l}-g_{m k} g_{j l}\right]+q\left[g_{m j} t_{k} t_{l}-g_{m k} t_{j} t_{l}+g_{k l} t_{m} t_{j}-g_{j l} t_{m} t_{k}\right]
$$

$p$ and $q$ are scalar functions. The first term describes constant curvature, the second one contains a vector field with $t_{k} t^{k}=1$.

The following identity holds:

$$
\begin{aligned}
b_{i}{ }^{m} R_{j k l m} & +b_{j}{ }^{m} R_{k i l m}+b_{k}{ }^{m} R_{i j l m}=q\left[g_{k l}\left(t_{j} b_{i}{ }^{m} t_{m}-t_{i} b_{j}{ }^{m} t_{m}\right)\right. \\
& \left.+g_{i l}\left(t_{k} b_{j}{ }^{m} t_{m}-t_{j} b_{k}{ }^{m} t_{m}\right)+g_{j l}\left(t_{i} b_{k}{ }^{m} t_{m}-t_{k} b_{i}{ }^{m} t_{m}\right)\right]
\end{aligned}
$$

Contraction with $g^{k l}$ gives: $-b_{i}{ }^{m} R_{j m}+b_{j}{ }^{m} R_{i m}=q(n-2)\left(t_{j} b_{i}{ }^{m} t_{m}-t_{i} b_{j}{ }^{m} t_{m}\right)$. Therefore, if $b$ commutes with the Ricci tensor and $n \neq 2$, the r.h.s. is zero and, by (17), $b$ is $R$-compatible. Then the Riemann tensor is pure and all Pontryagin forms vanish.

\section{StruCtures For Riemann COMPatibility}

Some differential structures are presented that yield Riemann compatibility. Of particular interest are geodesic mappings, which leave the condition for $R$ compatibility form-invariant, and generate $R$-compatible tensors.

6.1. Quasi Codazzi tensors. Let $b_{i j}$ be a symmetric tensor that solves the Codazzi condition deformed by a closed gauge field [22]:

$$
\left(\nabla_{j}-\beta_{j}\right) b_{k l}=\left(\nabla_{k}-\beta_{k}\right) b_{j l}
$$

The Codazzi deviation is $\mathscr{C}_{j k l}=\beta_{j} b_{k l}-\beta_{k} b_{j l}$, and $b$ is $R$-compatible.

Since $\beta_{i}=\nabla_{i} \xi$, the gauge field cancels for $b_{i j}=e^{\xi} b_{i j}^{\prime}$, where $b^{\prime}$ is a Codazzi tensor.

Of this type are Weakly $b$-symmetric manifolds, defined by the recurrency

$$
\nabla_{i} b_{k l}=A_{i} b_{k l}+B_{k} b_{i l}+D_{l} b_{i k}
$$

where $A, B$ and $D$ are covector fields. Eq.(18) is obtained for $\beta_{i}=A_{i}-B_{i}$, and $b$ is Riemann compatible if $A-B$ is closed.

Examples are: Weakly Ricci-symmetric manifolds, where $b_{i j}=R_{i j}$ [20, 21], Weakly and pseudo Z-symmetric manifolds, where $b_{i j}$ is a $Z$-tensor [21, 23]. Another example are manifolds with a recurrent generalized curvature tensor [20]: $\nabla_{i} K_{j k l}{ }^{m}=$ $A_{i} K_{j k l}{ }^{m}$, then $b_{k l}=: K_{k m l}{ }^{m} \neq 0$ has the form (19).

6.2. Pseudo-Ksymmetric manifolds. They are characterized by a generalized curvature tensor $K$ such that $([6,24])$

$$
\nabla_{i} K_{j k l}{ }^{m}=2 A_{i} K_{j k l}{ }^{m}+A_{j} K_{i k l}{ }^{m}+A_{k} K_{j i l}{ }^{m}+A_{l} K_{j k i}{ }^{m}+A^{m} K_{j k l i},
$$

The tensor $b_{j k}=: K_{j m k}{ }^{m}$ is symmetric. It is $R$-compatible if its Codazzi deviation $\mathscr{C}_{i k l}=A_{i} b_{k l}-A_{k} b_{i l}+3 A_{m} K_{i k l}{ }^{m}$ fulfills the II Bianchi identity. This is ensured by $A_{m}$ being concircular, i.e. $\nabla_{i} A_{m}=A_{i} A_{m}+\gamma g_{i m}$. 
6.3. Generalized Weyl tensors. A Riemannian manifold is a $(N C S)_{n} 28$ if the Ricci tensor satisfies $\nabla_{j} R_{k l}-\nabla_{k} R_{j l}=\frac{1}{2(n-1)}\left[g_{k l} \nabla_{j} R-g_{j l} \nabla_{k} R\right]$. As such, the Ricci tensor is the Weyl tensor, and the left hand side is its Codazzi deviation. This condition, by (12), is equivalent to $\nabla_{m} C_{j k l}{ }^{m}=0$.

This suggests a class of deviations of a symmetric tensor $b$ with $\mathscr{C}_{j k l}^{0}=0$ in (10):

$$
\mathscr{C}_{j k l}=\lambda_{j} g_{k l}-\lambda_{k} g_{j l}
$$

Proposition 6.1. $b$ is R-compatible if and only if $\lambda_{i}$ is closed.

Proof. Transvect (11) with $g^{k l}$ and obtain: $-b_{i}{ }^{m} R_{j m}+b_{j}{ }^{m} R_{i m}=(n-2)\left(\nabla_{i} \lambda_{j}-\right.$ $\left.\nabla_{j} \lambda_{i}\right)$. Then $b$ commutes with the Ricci tensor iff $\lambda$ is closed and, by the previous equation, $b$ is $R$-compatible.

An example is provided by spaces with

$$
\nabla_{j} b_{k l}=A_{j} g_{k l}+B_{k} g_{j l}+B_{l} g_{j k},
$$

where $\mathscr{C}_{j k l}=\lambda_{j} g_{k l}-\lambda_{k} g_{j l}$ with $\lambda_{j}=A_{j}-B_{j}$. Sinyukov manifolds [30] are of this sort, with $b_{i j}$ being the Ricci tensor itself.

6.4. Geodesic mappings. Riemann compatible tensors arise naturally in the study of geodesic mappings, i.e. mappings that preserve geodesic lines. Their importance arise from the fact that Sinyukov manifolds are $(N C S)_{n}$ manifolds and they always admit a nontrivial geodesic mapping.

Geodesic mappings preserve Weyl's projective curvature tensor [30. We show that they also preserve the form of the compatibility relation.

A map $f:\left(\mathscr{M}_{n}, g\right) \rightarrow\left(\mathscr{M}_{n}, \bar{g}\right)$ is geodesic if and only if Christoffel symbols are linked by $\bar{\Gamma}_{i j}^{k}=\Gamma_{i j}^{k}+\delta_{i}^{k} X_{j}+\delta_{j}^{k} X_{i}$ where, on a Riemannian manifold, $X$ is closed $\left(\nabla_{i} X_{j}=\nabla_{j} X_{i}\right)$. The condition is equivalent to:

$$
\nabla_{k} \bar{g}_{j l}=2 X_{k} \bar{g}_{j l}+X_{j} \bar{g}_{k l}+X_{l} \bar{g}_{k j}
$$

which has the form (21). The corresponding relation among Riemann tensors is

$$
\bar{R}_{j k l}^{m}=R_{j k l}{ }^{m}+\delta_{j}^{m} P_{k l}-\delta_{k}^{m} P_{j l}
$$

where $P_{k l}=\nabla_{k} X_{l}-X_{k} X_{l}$ is the deformation tensor. The symmetry $P_{k l}=P_{l k}$ is ensured by closedness of $X$.

Proposition 6.2. Geodesic mappings preserve $R$-compatibility

$$
b_{i m} \bar{R}_{j k l}{ }^{m}+b_{j m} \bar{R}_{k i l}{ }^{m}+b_{k m} \bar{R}_{i j l}{ }^{m}=b_{i m} R_{j k l}{ }^{m}+b_{j m} R_{k i l}{ }^{m}+b_{k m} R_{i j l}{ }^{m}
$$

where $b$ is a symmetric tensor.

Proof. Let's show that the difference of the two sides is zero. Eq.(23) gives:

$b_{i m}\left(\delta_{j}^{m} P_{k l}-\delta_{k}^{m} P_{j l}\right)+b_{j m}\left(\delta_{k}^{m} P_{i l}-\delta_{i}^{m} P_{k l}\right)+b_{k m}\left(\delta_{i}^{m} P_{j l}-\delta_{j}^{m} P_{i l}\right)$

$=b_{i j} P_{k l}-b_{i k} P_{j l}+b_{j k} P_{i l}-b_{j i} P_{k l}+b_{k i} P_{j l}-b_{k j} P_{i l}=0$

Since $\bar{g}$ is trivially $\bar{R}$-compatible (first Bianchi identity), form invariance implies:

Corollary 6.3. $\bar{g}$ is R-compatible.

Sinyukov [30] (see also [25, 12]) showed that a manifold admits a geodesic mapping if and only if there are a scalar field $\varphi$ and a symmetric non singular tensor $b_{i j}$ such that:

$$
\nabla_{k} b_{j l}=g_{k l} \nabla_{j} \varphi+g_{k j} \nabla_{l} \varphi .
$$


The Codazzi deviation of $b, \mathscr{C}_{j k l}=g_{k l} \nabla_{j} \varphi-g_{j l} \nabla_{k} \varphi$, has the form (20). Therefore $b$ is $R$-compatible.

\section{Generalized curvature tensors.}

Several results that are valid for the Riemann tensor with a Riemann compatible tensor, extend to generalized curvature tensors $K_{i j k l}$ (hereafter referred to as $K$ tensors) with a $K$-compatible symmetric tensor $b_{j k}$. The classical curvature tensors are $K$-tensors. The compatibility with the Ricci tensor is then examined.

Definition 7.1. A symmetric tensor $b_{i j}$ is $K$-compatible if

$$
b_{i m} K_{j k l}^{m}+b_{j m} K_{k i l}^{m}+b_{k m} K_{i j l}^{m}=0 .
$$

The metric tensor is always $K$-compatible, as (25) then coincides with the first Bianchi identity for $K$.

Proposition 7.2. If $K_{i j l m}$ is a $K$-tensor and $b_{k l}$ is $K$-compatible, then $\hat{K}_{i j k l}=$ : $K_{\text {ijrs }} b_{k}{ }^{r} b_{l}{ }^{s}$ is a $K$-tensor.

We quote without proof the extension of Derdzinski and Shen theorem for generalized curvature tensors 22]:

Theorem 7.3. Suppose that $K_{i j k l}$ is a $K$-tensor, and a symmetric $K$-compatible tensor $b_{i j}$ exists. Then, if $X, Y$ and $Z$ are three eigenvectors of the matrix $b_{r}{ }^{s}$ at a point $x$ of the manifold, with eigenvalues $\lambda, \mu$ and $\nu$, it is $X^{i} Y^{j} Z^{k} K_{i j k l}=0$ provided that both $\lambda$ and $\mu$ are different from $\nu$.

Proposition 7.4. If $b$ is $K$ - compatible, and $b$ commutes with a tensor $h$, then the symmetric tensor $K_{k l}^{\circ}=: K_{j k l m} h^{j m}$ commutes with $b$.

Proof. Multiply relation of $K$ compatibility for $b$ by $h^{k l}$. The last term vanishes for symmetry. The remaining terms give the null commutation relation.

In ref. 20] (prop.2.4) we proved that a generalization of Lovelock's identity (5) holds for certain $K$-tensors, that include all classical curvature tensors:

Proposition 7.5. Let $K_{j k l}{ }^{m}$ be a $K$-tensor with the property

$$
\nabla_{m} K_{j k l}{ }^{m}=\alpha \nabla_{m} R_{j k l}{ }^{m}+\beta\left(a_{k l} \nabla_{j}-a_{j l} \nabla_{k}\right) \varphi,
$$

where $\alpha, \beta$ are non zero constants, $\varphi$ is a real scalar function and $a_{k l}$ is a Codazzi tensor. Then:

$$
\begin{aligned}
& \nabla_{i} \nabla_{m} K_{j k l}{ }^{m}+\nabla_{j} \nabla_{m} K_{k i l}{ }^{m}+\nabla_{k} \nabla_{m} K_{i j l}{ }^{m} \\
& \quad=-\alpha\left(R_{i m} R_{j k l}{ }^{m}+R_{j m} R_{k i l}{ }^{m}+R_{k m} R_{i j l}{ }^{m}\right) .
\end{aligned}
$$

7.1. ABC curvature tensors. A class of $K$-tensors with the structure (26) are the $A B C$ curvature tensors. They are combinations of the Riemann tensor and its contractions ( $A, B, C$ are constants unless otherwise stated):

$$
\begin{array}{r}
K_{j k l}{ }^{m}=R_{j k l}{ }^{m}+A\left(\delta_{j}{ }^{m} R_{k l}-\delta_{k}{ }^{m} R_{j l}\right) \\
+B\left(R_{j}{ }^{m} g_{k l}-R_{k}{ }^{m} g_{j l}\right) \\
+C\left(R \delta_{j}{ }^{m} g_{k l}-R \delta_{k}{ }^{m} g_{j l}\right)
\end{array}
$$

The canonical curvature tensors are of this sort:

- Conformal tensor $C_{i j k l}: A=B=\frac{1}{n-2}, C=-\frac{1}{(n-1)(n-2)}$; 
- Conharmonic tensor $N_{i j k l}: A=B=\frac{1}{n-2}, C=0$;

- Projective tensor: $P_{i j k l}: A=\frac{1}{n-1}, B=C=0$;

- Concircular tensor: $\tilde{C}_{i j k l}: A-B=0, C=\frac{1}{n(n-1)}$.

Proposition 7.6. Let $K_{i j k l}$ be an $A B C$ tensor (A, B, C may be scalar functions) and $b_{i j}$ a symmetric tensor;

1) if $b$ is $R$-compatible then $b$ is $K$-compatible.

2) if $b$ is $K$-compatible and $B \neq \frac{1}{n-2}$ then $b$ is $R$-compatible.

Proof. The following identity holds for $A B C$ tensors and a symmetric tensor $b$ :

$$
\begin{aligned}
& \text { (29) } \quad b_{i m} K_{j k l}{ }^{m}+b_{j m} K_{k i l}{ }^{m}+b_{k m} K_{i j l}{ }^{m}=b_{i m} R_{j k l}{ }^{m}+b_{j m} R_{k i l}{ }^{m}+b_{k m} R_{i j l}{ }^{m} \\
& +B\left[g_{k l}\left(b_{i m} R_{j}{ }^{m}-b_{j m} R_{i}{ }^{m}\right)+g_{i l}\left(b_{j m} R_{k}{ }^{m}-b_{k m} R_{j}{ }^{m}\right)+g_{j l}\left(b_{k m} R_{i}{ }^{m}-b_{i m} R_{k}{ }^{m}\right)\right] .
\end{aligned}
$$

1 ) by theorem 4.3 , if $b$ is $R$-compatible then it commutes with the Ricci tensor, and $K$-compatibility follows.

2) if $b$ is $K$-compatible it commutes with $K_{i j}$. Contraction with $g^{k l}$ gives:

$$
b_{i m} K_{j}{ }^{m}-b_{j m} K_{i}{ }^{m}=\left(b_{i m} R_{j}{ }^{m}-b_{j m} R_{i}{ }^{m}\right)[1-B(n-2)],
$$

then, if $B \neq \frac{1}{n-2}, b$ commutes with the Ricci tensor and by (29) it is $R$-compatible.

Proposition 7.7. Let $K$ be an $A B C$ tensor with constant $A \neq 1$ and $B$. If

$$
\nabla_{i} \nabla_{m} K_{j k l}{ }^{m}+\nabla_{j} \nabla_{m} K_{k i l}{ }^{m}+\nabla_{k} \nabla_{m} K_{i j l}{ }^{m}=0
$$

then the Ricci tensor is $K$-compatible.

Proof. If $A$ and $B$ are constants, one evaluates

$$
\nabla_{m} K_{j k l}{ }^{m}=(1-A) \nabla_{m} R_{j k l}{ }^{m}+\frac{1}{2}(B+2 C)\left(g_{k l} \nabla_{j} R-g_{j l} \nabla_{k} R\right),
$$

Lovelock's identity (5) for the Riemann tensor implies

$$
\begin{aligned}
& \nabla_{i} \nabla_{m} K_{j k l}{ }^{m}+\nabla_{j} \nabla_{m} K_{k i l}{ }^{m}+\nabla_{k} \nabla_{m} K_{i j l}{ }^{m} \\
& \quad=-(1-A)\left(R_{i m} R_{j k l}{ }^{m}+R_{j m} R_{k i l}{ }^{m}+R_{k m} R_{i j l}{ }^{m}\right) .
\end{aligned}
$$

In the r.h.s. the Riemann tensor can be replaced by tensor $K$ by (29) written for the Ricci tensor.

Sufficient conditions are: $K$ is harmonic, $K$ is recurrent (with closed recurrency parameter, see eq.(3.13) in [20]). Note that prop. 7.7 remains valid for the Weyl's conformal tensor, which is traceless.

\section{Weyl-Compatibility and General Relativity}

In general relativity, the Ricci tensor is related to the energy-momentum tensor by the Einstein equation: $R_{j l}=\frac{1}{2} R g_{j l}+k T_{j l}$ with curvature $R=-2 k T /(n-2)$ $\left(T=T_{k}^{k}\right)$.

The contracted II Bianchi identity gives

$$
\nabla_{m} R_{j k l}{ }^{m}=k\left(\nabla_{k} T_{j l}-\nabla_{j} T_{k l}\right)+\frac{1}{2}\left(g_{j l} \nabla_{k} R-g_{k l} \nabla_{j} R\right) .
$$

Let $K$ be an $A B C$ tensor, with constant $A, B, C$. Its divergence (31) can be expressed in terms of the gradients of the energy momentum tensor $T_{i j}$. In the 
same way Einstein's equations and (32) give an equation which is local in the energy momentum tensor:

$$
\begin{aligned}
& \nabla_{i} \nabla_{m} K_{j k l}{ }^{m}+\nabla_{j} \nabla_{m} K_{k i l}{ }^{m}+\nabla_{k} \nabla_{m} K_{i j l}{ }^{m} \\
& \quad=-(1-A) k\left(T_{i m} K_{j k l}{ }^{m}+T_{j m} K_{k i l}{ }^{m}+T_{k m} K_{i j l}{ }^{m}\right) .
\end{aligned}
$$

The Weyl tensor $C_{j k l}{ }^{m}$ is the traceless part of the Riemann tensor, and it is an $A B C$ tensor. There are advantages in discussing General Relativity by taking the Weyl tensor as the fundamental geometrical quantity [2, 15, 7]. The first equation (31)

$$
\nabla_{m} C_{j k l}{ }^{m}=k \frac{n-3}{n-2}\left[\nabla_{k} T_{j l}-\nabla_{j} T_{k l}+\frac{1}{n-1}\left(g_{j l} \nabla_{k} T-g_{k l} \nabla_{j} T\right)\right]
$$

is reported in textbooks, as De Felice [7, Hawking Ellis [15, Stephani 31, and in the paper [2. Instead, a further derivation yields a Bianchi-like equation for the divergence, Eq.(33), which contains no derivatives of the sources

$$
\begin{aligned}
& \nabla_{i} \nabla_{m} C_{j k l}{ }^{m}+\nabla_{j} \nabla_{m} C_{k i l}{ }^{m}+\nabla_{k} \nabla_{m} C_{i j l}{ }^{m} \\
& \quad=-k \frac{n-3}{n-2}\left(T_{i m} C_{j k l}{ }^{m}+T_{j m} C_{k i l}{ }^{m}+T_{k m} C_{i j l}{ }^{m}\right) .
\end{aligned}
$$

It can be viewed as a condition for Weyl-compatibility for the energy momentum tensor.

In view of prop 7.4 and the previous equation, the following holds:

Proposition 8.1. If $T_{i j}$ is Weyl-compatible, the symmetric tensor $\stackrel{\circ}{k l}=: T^{j m} C_{j k l m}$ commutes with $T_{i j}$.

In 4 dimensions, given a time-like velocity field $u^{i}$, Weyl's tensor is projected in longitudinal (electric) and transverse (magnetic) tensorial components [2]

$$
E_{k l}=u^{j} u^{m} C_{j k l m}, \quad H_{k l}=\frac{1}{4} u^{j} u^{m}\left(\epsilon_{p q j k} C^{p q}{ }_{l m}+\epsilon_{p q j l} C_{k m}^{p q}\right)
$$

that solve equations that resemble Maxwell's equations with source. Therefore, the tensor $E_{k l}=\stackrel{\circ}{\mathrm{C}}_{k l}$ can be viewed as a generalized electric field. It coincides with the standard definition if $T_{i j}=(p+\rho) u_{i} u_{j}+p g_{i j}$ (perfect fluid). The generalized magnetic field is $H_{k l}=\frac{1}{4} T^{j m}\left(\epsilon_{p q j k} C^{p q}{ }_{l m}+\epsilon_{p q j l} C_{k m}^{p q}\right)$.

Proposition 8.2. If $T_{k l}$ is Weyl compatible then $H_{k l}=0$.

Proof. From the condition for Weyl compatibility we obtain $\epsilon_{i j k p}\left[T^{i m} C^{j k}{ }_{l m}+\right.$ $\left.T^{j m} C^{k i}{ }_{l m}+T^{k m} C^{i j}{ }_{l m}\right]=0$. The first and the second term are modified as follows:

$$
\begin{aligned}
& \epsilon_{i j k p} T^{i m} C^{j k}{ }_{l m}=\epsilon_{k i j p} T^{k m} C^{i j}{ }_{l m}=\epsilon_{i j k p} T^{k m} C^{i j}{ }_{l m} \\
& \epsilon_{i j k p} T^{j m} C^{k i}{ }_{l m}=\epsilon_{j k i p} T^{k m} C^{i j}{ }_{l m}=\epsilon_{i j k p} T^{k m} C^{i j}{ }_{l m} .
\end{aligned}
$$

Then, since the sum becomes $\epsilon_{i j k p} T^{k m} C^{i j}{ }_{l m}=0$, then the magnetic part of Weyl's tensor is zero.

\section{REFERENCES}

[1] Berger M. and Ebin D., Some characterizations of the space of symmetric tensors on a Riemannian manifold, J. Differential Geometry 3 (1969) 379-392.

[2] Bertschinger E. and Hamilton A. J. S., Lagrangian evolution of the Weyl tensor, Astroph. J. 435 1-7 (1994).

[3] Besse A. L., Einstein Manifolds, Springer (1987). 
[4] Bourguignon J. P., Les variétés de dimension 4 à signature non nulle dont la courbure est harmonique sont d'Einstein, Invent. Math. 63 n.2 (1981) 263-286.

[5] Chen B. Y. and Yano K., Hypersurfaces of a conformally flat space, Tensor N.S. 26 (1972) 318-322.

[6] De U. C., On weakly symmetric structures on Riemannian manifolds, Facta Universitatis, Series; Mechanics, Automatic Control and Robotics vol 3 n.14 (2003) 805-819.

[7] De Felice F. and Clarke C. J. S., Relativity on curved manifolds, Cambridge (2001).

[8] Derdzinski A., Some remarks on the local structure of Codazzi tensors, in Global differential geometry and global analysis, lecture notes n.838, Springer-Verlag (1981) 251-255.

[9] Derdzinski A., On compact Riemannian manifolds with harmonic curvature, Math. Ann. 259 (1982) 145-152.

[10] Derdzinski A. and Shen C. L., Codazzi tensor fields, curvature and Pontryagin forms, Proc. London Math. Soc. 47 n.3 (1983) 15-26.

[11] Ferus D., A remark on Codazzi tensors in constant curvature spaces, in global differential geometry and global analysis, lecture notes n.838, Springer-Verlag (1981) 257.

[12] Formella S., On some class of nearly conformally symmetric manifolds, Colloq. Math. 68 (1) (1995) 149-164.

[13] Gray A., Einstein-like manifolds which are not Einstein, Geom. Ded. 7 n.3 (1978) 259-280.

[14] Hamermesh M., Group theory and its application to physical problems, Dover reprint (1989).

[15] Hawking S. W. and Ellis G. F. R., The large scale structure of space time, Cambridge (1973)

[16] Kobayashi S. and Nomizu K, Foundations of differential geometry, Vol. 1, Interscience (1963) New York.

[17] Krupka D., The trace decomposition problem, Beitrage Algebra Geom 36 n.2, 303-315 (1995).

[18] Lovelock D. and Rund H., Tensors, differential forms and variational principles, reprint Dover Ed. (1988).

[19] Maillot H., Sur les variétés Riemanniennes à operateur de courbure pur, C. R. Acad. Sci. Paris Ser. A 278 (1974) 1127-1130.

[20] Mantica C. A. and Molinari L. G., A second order identity for the Riemann tensor and applications, Colloq. Math. 122 n.1 (2011) 69-82; arXiv:0802.0650 [math.DG].

[21] Mantica C. A. and Molinari L. G., Weakly Z-symmetric manifolds, Acta Math. Hung. 135 n.1-2 (2012) 80-96; arXiv:1102.5340 [math.DG].

[22] Mantica C. A. and Molinari L. G., Extended Derdzinski-Shen theorem for curvature tensors, submitted to Colloquium Mathematicum (2012), arXiv:1101.4157 [math.DG].

[23] Mantica C. A. and Suh J. Y., Pseudo Z symmetric Riemannian manifolds with harmonic curvature tensors, Int. J. Geom. Meth. Mod. Phys. 9 n.1, 1250004 (21 pages) (2012).

[24] Mantica C. A. and Suh J. Y., The closedness of some generalized 2-forms on a Riemannian manifold I, Publ. Math. Debrecen (2012).

[25] Mikes J., Geodesic Mappings of affine-connected and Riemannian spaces, Journal of Mathematical Science 78 (3) 311-334 (1996).

[26] Nakahara M., Geometry, Topology and Physics, Adam Hilger, Bristol (1990)

[27] Petersen P., Riemannian Geometry, Springer (2006).

[28] Roter W., On a generalization of conformally symmetric metrics, Tensor (NS) 46 278-286 (1987).

[29] Simon U., Codazzi tensors, in global differential geometry and global analysis, lecture notes n.838, Springer-Verlag (1981), 289-296.

[30] Sinyukov N. S., Geodesic mapping of Riemannian spaces, Nauka, Moscow (1979).

[31] Stephani H., Kramer D., MacCallum M., Hoenselaers C. and Hertl E., Exact Solutions of Einstein's Field Equations, Cambridge University Press, 2nd ed. (2003).

C. A. Mantica: I.I.S. Lagrange, Via L. Modignani 65, 20161, Milano, Italy - L. MoliNari (CORResponding author): Physics Department, Universitá degli Studi di Milano and I.N.F.N. Sez. Milano, Via Celoria 16, 20133 Milano, Italy.

E-mail address: carloalberto.mantica@libero.it, luca.molinari@unimi.it 